\title{
A Clinico-pathological Study of Cases of Pemphigus
}

\author{
Dr. Jayesh Shah ${ }^{1}$, Dr. Devendra Parmar ${ }^{2}$ \\ ${ }^{1}$ Professor, Department of Skin, Gujarat Adani Institute of Medical Sciences, Bhuj, Gujarat, India \\ ${ }^{2}$ Associate Professor, Department of Skin, Gujarat Adani Institute of Medical Sciences, Bhuj, Gujarat, India
}

\begin{abstract}
In pemphigus, autoantibodies form against desmoglein. Desmoglein forms the "glue" that attaches adjacent epidermal cells via attachment points called desmosomes. When autoantibodies attack desmogleins, the cells become separated from each other and the epidermis becomes "unglued", a phenomenon called acantholysis. This causes blisters that slough off and turn into sores. In some cases, these blisters can cover a significant area of the skin. Histopathological evaluation of biopsies received from the department of Dermatology. Results: A total number of 400 skin biopsies were received, out of which 32 cases were of the pemphigus group of diseases. Pemphigus vulgaris accounted for $81 \%$ (26) of cases and pemphigus vegetans accounted for $6 \%$ (3) of cases. The less common types were pemphigus erythematosus and IgA pemphigus constituting $6 \%$ (3) of the cases. Pemphigus vulgaris formed the most common variant.
\end{abstract}

Keywords: Pemphigus, Vulgaris, Cinicopathological

\section{Introduction}

The autoimmune bullous dermatoses fall into 2 main groups: diseases of the dermoepidermal junction, which are due to abnormalities at the interface between the dermis and the epidermis (of which pemphigoid is one example) and intraepithelial dermatoses, which include the various forms of pemphigus. ${ }^{1}$ Pemphigus results from circulating immunoglobulin $\mathrm{G}$ (IgG) antibodies directed against desmosomes; these antibodies interfere with keratinocyte adhesion. Acantholysis occurs, resulting in the formation of bullae. ${ }^{2}$ There are 6 main types of pemphigus and their classification is based on the anatomic features of the lesion and the target antigens recognized by the autoantibodies. ${ }^{3}$

Eighty percent of patients with pemphigus have pemphigus vulgaris (PV). The annual incidence is estimated as one case per million populations, but the condition is more common among Ashkenazi Jews and people of Mediterranean descent because of an association with certain human leukocyte antigen haplotypes. ${ }^{4}$

Pemphigus is a rare autoimmune disorder with intraepidermal bullous lesions which affect in particular the oral, genital or ocular mucosa and the epidermis. ${ }^{5}$ This condition typically affects people between the ages of 50 and 60 and is generally evenly distributed between the sexes. $\mathrm{PV}$, the most common form of pemphigus in Europe, represents $80 \%$ of all cases. The incidence of PV is $0.1-0.5$ per 100,000 population in the United States. The initial lesions are often insidious and localized. The mouth is affected by persistent, painful ulcers and a burning sensation, which affects the appetite. ${ }^{3}$

Although a few studies are available on this relatively uncommon disease, apart from the classical histopathological features, this study highlights some interesting and rarely encountered histological findings.

\section{Materials \& Method}

A total number of 400 skin biopsies were performed during the study period of 2 years, out of which 32 cases were of the pemphigus group of skin diseases which accounted for $8 \%$ of the cases. The histopathological evaluation of biopsies was done in the Department of Pathology, in the same hospital. The biopsy specimens were stored in $10 \%$ formalin for the fixation of the tissues. The biopsy specimens were subjected to routine processing and the paraffin embedding. Multiple serial sections were taken for each biopsy specimen and all were stained with hematoxylin and eosin. Immunofluorescence advanced techniques was done wherever possible in the specimen.

\section{Results}

Out of the 32 cases of the pemphigus group of diseases, pemphigus vulgaris accounted for $81 \%$ (26) of the cases and pemphigus vegetans accounted for $9.01 \%$ (3) of the cases. The less common cases were pemphigus erythematosus and IgA pemphigus constituting $9.1 \%$ ( 3 ) of the cases.

Table 1: Distribution of lesions of pemphigus

\begin{tabular}{|l|l|l|l|}
\hline SI. No. & \multicolumn{1}{|c|}{ Disorder } & No. of Cases & \multicolumn{1}{|c|}{$\%$} \\
\hline 1 & Pemphigus Vulgaris & 26 & 81 \\
\hline 2 & Pemphigus Vegetans & 3 & 9 \\
\hline 3 & Pemphigus Erythematous & 2 & 6.25 \\
\hline 4 & IgA Pemphigus & 1 & 3.12 \\
\hline Total & & 32 & $100 \%$ \\
\hline
\end{tabular}

A majority of the lesions in pemphigus vulgaris were distributed in the oral cavity- $20(62.5 \%)$, followed by trunk$4(12.5 \%)$ and extremities - $6(18.75 \%)$, back $-1(3 \%)$ and axilla- $1(3 \%)$. Most of them were flaccid bullae (85.89\%) which were situated on a non erythematous base. A majority of pemphigus vulgaris patients were between $30-60$ years of age. IgA pemphigus and pemphigus vegetans were found in the 5th and 6th decades of life, respectively. The male to female ratio of pemphigus was nearly $1: 1$. The 


\section{International Journal of Science and Research (IJSR) \\ ISSN (Online): 2319-7064 \\ Index Copernicus Value (2013): 6.14 | Impact Factor (2014): 5.611}

histopathological features observed in 20 cases of pemphigus vulgaris were, a suprabasal split in $94.4 \%$ (28) of the cases. Neutrophils, eosinophils and acantholytic cells in clusters were observed inside the bulla in $94.4 \%$ (28) of the cases. Adjacent epidermis showed acantholysis in 11.3\% (6) and spongiosis in $61.1 \%$ (18) of the cases. Acantholysis of the follicular sheath were seen in $22.2 \%$ (6) cases and prominent villi in $27.5 \%$ (5) cases. Tzanck smear was done in all the cases. All the cases showed the presence of acantholytic cells and mixed inflammatory cells which were composed of eosinophils and lymphocytes. Both the cases of pemphigus vegetans showed hyperkeratosis, papillomatosis and acanthosis. Suprabasal lacunae, with a few acantholytic cells. One case each of pemphigus erythematosus and IgA pemphigus were seen. Pemphigus erythematosus showed subcorneal bulla filled with neutrophils, eosinophils and acantholytic cells. Spongiosis of the adjacent epidermis and follicular sheath, along with acanthosis, were also noted. IgA pemphigus had both subcorneal and intraepidermal bullae with neutrophils and fibrin. The adjacent epidermis showed spongiosis.

Direct immunofluorescence was done in 8 cases of pemphigus vulgaris, which showed intercellular lace like pattern with $\mathrm{IgG}$ and $\mathrm{C} 3$ in 4 cases and only $\operatorname{IgG}$ in 4 cases.

\section{Discussion}

The mean age of patients with bullous pemphigoid in the study is similar to other published data. However, with the exception of one regional study, all studies were hospital based; therefore, although they may have had excellent validity, they may be less representative of the disease in the population. The mean age of patients with pemphigus vulgaris in It has been was much higher than previous reports. However, no data on the epidemiology of pemphigus vulgaris in western Europe have been published and all of the studies are from hospital centres in the form of either cross sectional surveys or case series; these studies are unlikely to give reliable population based statistics. ${ }^{6}$

The incidence of bullous pemphigoid in It has been is similar to that described by Garcia-Doval and colleagues, the incidence of pemphigus vulgaris is within the range of the previous papers, and the sex distribution is also similar.The increasing trend in incidence of bullous pemphigoid has not been previously shown, but no study has looked at incidence of bullous pemphigoid prospectively over time. ${ }^{6}$ A possible explanation is increased ascertainment, but if this is the case the increasing trend has not levelled off. The number of consultant dermatologists in the UK increased by $39 \%$ between 1998 and 2006. This may have contributed to increased ascertainment but is unlikely to explain completely the increased incidence because of the acute, severe presentations of these disorders. ${ }^{6}$

It has been shows that rates of bullous pemphigoid seem to be increasing over time. Rates of pemphigus vulgaris are increasing to a lesser degree despite the facts that both disorders are more common with increasing age and that the multivariate analysis was adjusted for age. ${ }^{7}$ Possible explanations include ascertainment bias, misclassification, and a true increase in incidence. If the last of these is true, further investigation is warranted into the reasons for this, as it may provide further insight into the causes of the disorders. The lack of a socioeconomic gradient and major geographical variation argues against the environment playing an important role in causing the diseases. ${ }^{6}$

It has been has shown high mortality associated with these diseases; rates were twice that in the general population in patients with bullous pemphigoid and three times as high as in the general population in those with pemphigus vulgaris. ${ }^{8}$ With the increasing age of our population, the morbidity and mortality from these diseases will increase. Previous measures have underestimated the burden of these diseases, in terms of both incidence and risk of death. ${ }^{9}$

An accurate histological diagnosis is essential for the therapy. The association of clinical and histopathological features helps to arrive at the diagnosis in most cases. The study also revealed additional features which are not encountered normally, like the extrusion of adnexal structures into the bulla cavity and the extension of spongiosis and acantholysis into the adnexal epithelium. ${ }^{10}$ Analysis of subtle light microscopic features, apart from the classical diagnostic features, threw light on the diagnosis of difficult cases. Immunofluorescence study helped in confirming the diagnosis where histopathology and clinical features alone were inconclusive

\section{References}

[1] Crowson AN, Magro C: The cutaneous pathology of lupus erythematosus: a review. Journal of cutaneous pathology 2001, 28:1-23.

[2] Stanley JR, Amagai M: Pemphigus, bullous impetigo, and the staphylococcal scalded-skin syndrome. New England Journal of Medicine 2006, 355:1800-10.

[3] Lagha NB, Poulesquen V, Roujeau J, Alantar A, Maman L: Pemphigus vulgaris: a case-based update. Journal-Canadian Dental Association 2005, 71:667.

[4] Harman K, Seed P, Gratian M, Bhogal B, Challacombe $\mathrm{S}$, Black M: The severity of cutaneous and oral pemphigus is related to desmoglein 1 and 3 antibody levels. British Journal of Dermatology 2001, 144:77580.

[5] Kneisel A, Hertl M: Autoimmune bullous skin diseases. Part 1: clinical manifestations. JDDG: Journal der Deutschen Dermatologischen Gesellschaft 2011, 9:84457.

[6] Langan S, Smeeth L, Hubbard R, Fleming K, Smith C, West J: Bullous pemphigoid and pemphigus vulgarisincidence and mortality in the UK: population based cohort study. Bmj 2008, 337.

[7] Kirtschig G, Khumalo NP: Management of bullous pemphigoid. American journal of clinical dermatology 2004, 5:319-26.

[8] Knudson RM, Kalaaji AN, Bruce AJ: The management of mucous membrane pemphigoid and pemphigus. Dermatologic therapy 2010, 23:268-80.

[9] Yusuf S, Reddy S, Ôunuu S, Anand S: Global burden of cardiovascular diseases part I: general considerations, the epidemiologic transition, risk factors, and impact of urbanization. Circulation 2001, 104:2746-53.

[10] MURTHY DN: THROUGH PROPER CHANNEL.

\section{Volume 4 Issue 12, December 2015}

